\title{
THEOREM ON THE UNION OF TWO TOPOLOGICALLY FLAT CELLS OF CODIMENSION 1 IN $\mathbb{R}^{n}$
}

\author{
A. V. CHERNAVSKY \\ Received 26 June 2005; Accepted 1 July 2005
}

In this paper we give a complete and improved proof of the "Theorem on the union of two $(n-1)$-cells." First time it was proved by the author in the form of reduction to the earlier author's technique. Then the same reduction by the same method was carried out by Kirby. The proof presented here gives a more clear reduction. We also present here the exposition of this technique in application to the given task. Besides, we use a modification of the method, connected with cyclic ramified coverings, that allows us to bypass referring to the engulfing lemma as well as to other multidimensional results, and so the theorem is proved also for spaces of any dimension. Thus, our exposition is complete and does not require references to other works for the needed technique.

Copyright (c) 2006 A. V. Chernavsky. This is an open access article distributed under the Creative Commons Attribution License, which permits unrestricted use, distribution, and reproduction in any medium, provided the original work is properly cited.

\section{Notations and statement of the result}

Specify the standard coordinate system $O x^{1} \cdots x^{n}$ with the origin $O$ and coordinate axes $O x^{i}$ in the space $\mathbb{R}^{n}$. The coordinate planes $O x^{1} \cdots x^{i}$ will be identified with $\mathbb{R}^{i}$. The unit disc in $\mathbb{R}^{i}$ is denoted by $B^{i}$. The semispaces $x^{n-1} \geq 0$ and $x^{n-1} \leq 0$ are denoted as $\mathbb{R}_{+}^{n}$ and $\mathbb{R}_{-}^{n}$, respectively, while semiplanes $\mathbb{R}_{+}^{n} \cap \mathbb{R}^{n-1}$ and $\mathbb{R}_{-}^{n} \cap \mathbb{R}^{n-1}$ as $\mathbb{R}_{+}^{n-1}$ and $\mathbb{R}_{-}^{n-1}$, respectively. Semidiscs $B^{n-1} \cap \mathbb{R}_{+}^{n-1}$ and $B^{n-1} \cap \mathbb{R}_{-}^{n-1}$ are denoted by $B_{+}^{n-1}$ and $B_{-}^{n-1}$, respectively.

We will say that an embedding $q: B^{i} \rightarrow M^{n}$ of an $i$-disc in a topological $n$-manifold without boundary is topologically flat if one can extend it to an embedding in $M^{n}$ of its neighborhood in $\mathbb{R}^{n}$. It is known that a topologically flat embedding of a disc into $\mathbb{R}^{n}$ is extendable to a homeomorphism of $\mathbb{R}^{n}$ onto itself. An embedding of a submanifold is locally flat if every point has a neighborhood in it that is homeomorphic to a disc and the embedding on this disc is topologically flat. Any locally flat embedding of a disc is topologically flat (see, e.g., [8]).

Theorem 1.1. Let an embedding $q: B^{n-1} \rightarrow \mathbb{R}^{n}$ be given, whose restrictions to both semidiscs $B_{+}^{n-1}$ and $B_{-}^{n-1}$ are topologically flat. Then $q$ is topologically flat. 
We will denote the restriction of $q$ onto $B_{ \pm}^{n-1}$ as $q_{ \pm}$, respectively.

Notice an important corollary to this theorem (first time mentioned by Cantrell [1]) that in the case $n \geq 4$ for embedding of an $(n-1)$-manifold into an $n$-manifold there are no isolated points where the condition of locally flatness is destroyed. If $n=3$, it is not the case. The reason for this difference is the fact that for $n \geq 4$ an isolated singularity cannot exist on the boundary of an $(n-1)$-submanifold, and this is derived from the fact that in the former dimensions the arcs with only one singularity do not exist, (see $[5,6]$ ) while it is well known that in the dimension 3 they do exist.

The proof of this theorem is based on a series of lemmas using the constructions of some elementary homeomorphisms described in Section 2. Here we introduce some notations.

Denote by $\Pi_{\alpha}$ the semiplane, bounded by subspace $R^{n-2}$ and having the angle of $\alpha$ radians with $\mathbb{R}_{+}^{n-1}=\Pi_{0}$. $\left(\Pi_{-\pi}=\Pi_{\pi}=\mathbb{R}_{-}^{n-1}\right.$.) $Q[\alpha, \beta], \alpha<\beta$ will denote a closed domain between $\Pi_{\alpha}$ and $\Pi_{\beta}\left(Q[\alpha, \beta]=\cup_{\alpha \leq \gamma \leq \beta} \Pi_{\gamma}\right), Q(\alpha, \beta)$ denotes the interior of $Q[\alpha, \beta]$.

For a point $z \in \mathbb{R}^{n}$ we denote by $x_{z}$ its projection onto $\mathbb{R}^{n-2}$ and by $y_{z}$ its projection onto $\mathbb{R}^{n-1}$.

Consider the system of 2-planes $P_{x}, x \in \mathbb{R}^{n-2}$ orthogonal to $\mathbb{R}^{n-2}$ at the corresponding points $x$. Consider also in every plane $P_{x}$ an orthonormal coordinate system with the origin $x$ and axes $x s$ and $x t$, the former is parallel and codirected with the axe $O x^{n-1}$, and the latter is parallel and codirected with the axe $O x^{n}, s$ and $t$ have the meaning of coordinate parameters. For a point $z \in \mathbb{R}^{n}$ we denote by $s_{z}$ and $t_{z}$ its coordinates in the plane $P_{x_{z}}$. At last, $C_{x}(r)$ will denote a circle with the radius $r$ in the plane $P_{x}$ centred at $x$. For a point $z \in \mathbb{R}^{n}$ we denote by $r_{z}$ its distance from $\mathbb{R}^{n-2}$, that is, the radius $r$ of a circle $C_{x_{z}}(r)$ passing through $z$.

\section{Preliminary statements}

The following two statements will help us to construct some elementary homeomorphisms of $\mathbb{R}^{n}$ that send every circle $C_{x}(r)$ onto itself piecewise linearly.

Statement 2.1. Let for some $\alpha$ a closed subset $M \subset \mathbb{R}^{n}$ be given such that in some neighborhood of $B^{n-2}$ it does not intersect $\Pi_{\alpha} \backslash \mathbb{R}^{n-2}$ and lies on one side of $\Pi_{\alpha}$ (i.e., in $Q(\alpha, \alpha+\pi)$ or in $Q(\alpha-\pi, \alpha))$.

Then there exists a function $\varepsilon(z)>0, z \in \Pi_{\alpha} \backslash \mathbb{R}^{n-2}$ (possibly in a smaller neighborhood of $B^{n-2}$ ), that is continuous, tending to zero as $z$ is tending to a point in $\mathbb{R}^{n-2}$, and such that for any circle $C_{x}(r)$ in this neighborhood its arc with the length $\varepsilon(z)$, having an end in $z \in \Pi_{\alpha}$ and lying on one side of $\Pi_{\alpha}$ as $M$, does not intersect $M$.

The construction of $\varepsilon(z)$ is standard and evident, so that it may be omitted.

For $z \in \Pi_{\alpha}$ consider arcs of the circles $C_{x_{z}}\left(r_{z}\right)$ having one end at $z$ and the length $\varepsilon(z)$, where this function is chosen according to Statement 2.1 for some set $M$, the arcs are taken on one side of $\Pi_{\alpha}$, as $M$. The surface, described by the second ends of these arcs (i.e., not on $\Pi_{\alpha}$ ) will be called the fence separating $M$ from $\Pi_{\alpha}$.

Note that every circle $C_{x}$ (sufficiently close to $B^{n-2}$ ) intersects every $\Pi_{\alpha}$ and every fence exactly one time. 
Statement 2.2. Let four sets $A, B, C, D$ in a neighborhood of $B^{n-2}$ be given so that each of them is either a $\Pi_{\alpha}$ or a fence, and the points of intersections of $B$ and $C$ with any circle $C_{x}(r)$ in this neighborhood are located between points of intersection of this circle with the sets $A$ and $D$. Then there exists a homeomorphism of $\mathbb{R}^{n}$, identical outside a neighborhood of $B^{n-2}$ and outside the domain between $A$ and $D$ (containing $B$ and $C$ ), that sends $B$ into $C$ in a smaller neighborhood.

For the proof it is sufficient to construct a homeomorphism on every circle $C_{x}(r)$ in a small neighborhood of $B^{n-2}$ that maps linearly the arc between $A$ and $B$ into the arc between $A$ and $C$ and simultaneously the arc between $B$ and $D$ into the arc between $C$ and $D$, such that it is identical on the second arc between $A$ and $D$. In some larger neighborhood one can continuously reduce this homeomorphism to the identity.

The homeomorphisms constructed as in this proof will be called arcwise. Note that arcwise homeomorphisms are naturally isotopic to the identity.

Before turning to our lemmas, let us introduce the following definitions.

Defintions 2.3. An embedding $\gamma: \Pi_{\alpha} \rightarrow \mathbb{R}^{n}$, being identity on $\mathbb{R}^{n-2}$, touches $\Pi_{\beta}$ at points of $B^{n-2}$ if for every $\varepsilon>0$ one can find $\delta>0$ so that $\gamma \Pi_{\alpha} \cap O_{\delta}\left(B^{n-2}\right) \subset Q[\beta-\varepsilon, \beta+\varepsilon]$.

Analogously, a sequence of points $z_{n} \in \mathbb{R}^{n}$ touches $\Pi_{\alpha}$ at a point $x \in B^{n-2}$ if for every $\varepsilon>0$ there exists $n_{\varepsilon}$ such that $z_{n} \in Q[\beta-\varepsilon, \beta+\varepsilon] \cap O_{\varepsilon}(x)$ for all $n>n_{\varepsilon}$.

\section{Lemmas}

Lemma 3.1. Let an embedding $p_{1}: B^{n} \backslash\left(B_{-}^{n-1} \backslash B^{n-2}\right) \rightarrow \mathbb{R}^{n}$ be identical on $B^{n-2}$, and for every $\alpha \in[-\pi+\pi / 4, \pi-\pi / 4]$ the set $p_{1}\left(\Pi_{\alpha}\right)$ touches $\Pi_{\alpha}$. Let also $p_{1} B_{+}^{n-1} \subset \mathbb{R}_{+}^{n}$.

Then the cell $B_{-}^{n-1} \cup p_{1} B_{+}^{n-1}$ is embedded topologically flat, that is, there is a homeomorphism $\bar{p}_{1}$ of $\mathbb{R}^{n}$ that maps $B^{n-1}$ onto $B_{-}^{n-1} \cup p_{1} B_{+}^{n-1}$. (The tangency of $\Pi_{\alpha}$ for $\alpha \in$ $Q(-\pi / 2,+\pi / 2)$ is not essential and has only a technical role.)

Proof. First we will construct a mapping $w: \mathbb{R}^{n} \rightarrow \mathbb{R}^{n}$, that orthogonally projects $B_{-}^{n-1}$ onto $B^{n-2}$, is homeomorphic outside $B_{-}^{n-1}$, and is identical on $\mathbb{R}_{+}^{n}$. Under the given conditions it is clear that the composition $w^{-1} \pi w$ coincides with $p_{1}$ on $B_{+}^{n-1}$. At the same time it occurs that this composition can be extended identically on $B_{-}^{n-1}$. The obtained extension is the homeomorphism $\bar{p}_{1}$ we are looking for.

The beginning of this construction of $w$ is determined by the requirements that $w=1$ on $\mathbb{R}_{+}^{n}$ and $w(y)=x_{y}$ for $y \in B_{-}^{n-1}$. Extend $w$ identically to the points $y \in \mathbb{R}_{-}^{n-1}$ whose projections $x_{y}$ onto $\mathbb{R}^{n-2}$ are situated outside $B^{n-2}$. If $x_{y} \in B^{n-2}$ and $y \in \mathbb{R}_{-}^{n-1} \backslash B_{-}^{n-1}$, we take as $w(y)$ the point that is obtained from $y$ by the shift along the direction of the axe $O x^{n-1}$ in the distance equal to the intersection segment of $B_{-}^{n-1}$ with the axe $x_{y} s$ in $P_{x_{y}}$. Thus we have constructed $w$ on the space $\mathbb{R}^{n-1}$.

For every point $y \in \mathbb{R}_{-}^{n-1}$, we denote by $L_{y}$ the straight line going through $y$ and being parallel to the axe $O x^{n}$. If $x_{y}$ lies outside $B^{n-2}$, set $w=1$ on $L_{y}$.

Let $y \in B^{n-1}$. Define that $w$ sends $L_{y}$ isometrically into the union of two rays in $P_{x_{y}}$ starting at the point $x_{y} \in B^{n-2}$ with the angle $\alpha= \pm\left(\pi / 2-\pi / 4 \cdot s_{y}\right)$ with respect to the axe $x_{y} s\left(s_{y}<0\right.$ is the coordinate of $y$ in $\left.P_{x_{y}}\right)$.

Notice that $\alpha \rightarrow \pi / 2$, when $s_{y} \rightarrow 0$, that is, $y \rightarrow x_{y}$. 
If $x_{y} \in B^{n-2}$ and $y \in \mathbb{R}_{-}^{n-1} \backslash B^{n-1}$, then $w$ sends $L_{y}$ isomorphically to the pair of rays in $P_{x_{y}}$ starting at the point $w(y)$ with the angles $\alpha= \pm\left(\pi / 2-\pi / 4 \cdot s_{y^{\prime}}\right)$, where $y^{\prime}$ is an intersection of the half-axe $x_{y} s \cap \mathbb{R}_{-}^{n}$ with the boundary of $B^{n-1}$.

Now $w$ is well posed on the entire $\mathbb{R}^{n}$; it is continuous and identical on $\mathbb{R}_{+}^{n}$ and outside $\cup_{x \in B^{n-2}} P_{x}$. Also $w$ retracts $B_{-}^{n-1}$ onto $B^{n-2}$ by the orthogonal projection and it is homeomorphic outside $B_{-}^{n-1}$.

It remains to note that a sequence of points $z_{n}$ tends to a point $y \in B_{-}^{n-1}$ if and only if $w\left(y_{n}\right)$ tends to $x_{y}$, touching $\Pi_{\alpha} \cup \Pi_{-\alpha}$, where $\alpha$ is chosen according to the point $y$ as above, that is, $\alpha_{y}= \pm\left(\pi / 2-\pi / 4 \cdot s_{y}\right)$.

Indeed, take a spherical neighborhood $V_{\varepsilon}$ with radius $\varepsilon>0$ of a point $x_{y}$ in the plane $x^{n-1}=0$ and consider the set $W_{\varepsilon}$ of points $z \in \mathbb{R}_{-}^{n}$ that are projected to $V_{\varepsilon}$. Let $U_{\varepsilon}(y)$ be the intersection of $W_{\varepsilon}$ with the domain between two planes, being parallel to $x^{n-1}=0$ and located on different sides of $y$ in the distance $\varepsilon$. Let $U_{\varepsilon}^{\prime}\left(x_{y}\right)$ be the intersection of $W_{\varepsilon}$ with $Q\left(\alpha_{y}-\pi / 2 \cdot \varepsilon, \alpha_{y}+\pi / 2 \cdot \varepsilon\right) \cup Q\left(-\alpha_{y}-\pi / 2 \cdot \varepsilon,-\alpha_{y}+\pi / 2 \cdot \varepsilon\right)$. Then for every $\varepsilon^{\prime}>0$ one can find a $\varepsilon>0$ such that $w\left(U_{\varepsilon}(y)\right) \subset U_{\varepsilon^{\prime}}^{\prime}$, and, conversely, for every $\varepsilon>0$ one can find a $\varepsilon^{\prime}$ such that $w\left(U_{\varepsilon}(y)\right) \supset U_{\varepsilon^{\prime}}^{\prime}\left(x_{y}\right)$. Hence the sequence of points $z_{n} \in \mathbb{R}^{n}$ tends to $y \in \operatorname{Int} B_{-}^{n-1}$ if and only if $w\left(z_{n}\right)$ tends to $x_{y}$ and touches $\Pi_{\alpha_{y}} \cup \Pi_{-\alpha_{y}}$.

A sequence $z_{n}$ tends to $y \in \partial B_{-}^{n-1} \backslash B^{n-2}$ if and only if $w z_{n} \rightarrow x_{y}$ and for every $\varepsilon>0$ there exists $n_{0}$ such that for $n>n_{0}$ all $z_{n}$ are located outside $Q(-\pi / 2-\pi / 4+\varepsilon,+\pi / 2+$ $\pi / 4-\varepsilon)$. It is clear that the same property is fulfilled for the sequence $h w\left(z_{n}\right)$.

This proves that the homeomorphism $\tilde{p}_{1}=w^{-1} p_{1} w$ is extended identically to $B_{-}^{n-1}$, as what was in demand. The constructed homeomorphism $\tilde{p}_{1}$ coincides with the given $p_{1}$ on $B_{+}^{n-1}$ and is identical on $B_{+}^{n-1}$. Thus, the union of cells $B_{-}^{n-1} \cup p_{1} B_{+}^{n-1}=\bar{p}_{1} B^{n}$ is embedded locally flat at least at the points of $B^{n-1} \backslash \partial B^{n-2}$. But then one can easily construct a homeomorphism of the whole space that sends $B_{-}^{n-1} \cup p_{1} B_{+}^{n-1}$ into $B_{-}^{n-1}$. It is a standard construction (see [3]), which we leave as an exercise. So, the embedding of $B_{-}^{n-1} \cup p_{1} B_{+}^{n-1}$ is topologically flat.

Lemma 3.2. The assertion of Lemma 3.1 is true for the embedding $p_{2}: Q[-\pi / 2, \pi / 2] \rightarrow$ $Q[-\pi / 2, \pi / 2]$, for which $p_{2} \Pi_{\alpha}$ touches $\Pi_{\alpha}$ with $\alpha \in(-\pi / 2, \pi / 2), p_{2} B_{+}^{n-1} \subset Q(-\pi / 4, \pi / 4)$.

Proof. Construct the arc homeomorphism $\rho: Q[-\pi, \pi] \rightarrow Q[-\pi / 2, \pi / 2]$, identical on $Q[-\pi / 4, \pi / 4]$, that sends linearly the arc of each circle $C_{x}(r)$ between the points of its intersections with $\Pi_{\pi}$ and $\Pi_{\pi / 4}$ to the arc between its intersections with $\Pi_{\pi / 2}, \Pi_{\pi / 4}$, and, analogously, sends the arc between $\Pi_{-\pi}$ and $\Pi_{-\pi / 4}$ to the arc between $\Pi_{\pi / 2}$ and $\Pi_{-\pi / 4}$. It is clear that touching $\Pi_{\alpha}$ is transformed into touching $\rho \Pi_{\alpha}$. Then, the hypothesis of Lemma 3.1 is satisfied for $\rho^{-1} p_{2} \rho$ that coincides with $p_{2}$ on $B_{+}^{n-2}$. Thus, the embedding of the cell $B_{-}^{n-1} \cup p_{2} B_{+}^{n-2}$ is topologically flat.

Lemma 3.3. Let an embedding $p_{3}: \mathbb{R}_{+}^{n} \rightarrow \mathbb{R}_{+}^{n}$ be given in some neighborhood of $B^{n-2}$, where the images of $\Pi_{-\pi / 2}, \Pi_{\pi / 2}$, and two more semiplanes $\Pi_{\alpha},-\pi / 2<\alpha<\pi / 2$ (let them be for definiteness $\Pi_{-\pi / 4}$ and $\left.\Pi_{0}\right)$ touch their preimages: $\Pi_{-\pi / 2}, \Pi_{\pi / 2}, \Pi_{-\pi / 4}, \Pi_{0}$ at the points of $B^{n-2}$.

Then there exists an isotopy $\phi_{t}: \mathbb{R}^{n} \rightarrow \mathbb{R}^{n}$, identical outside $p_{3} \mathbb{R}_{+}^{n}$ and outside a given neighborhood of $B^{n-2}$, such that $\phi_{0}=1$ and $\bar{p}_{3} \Pi_{\alpha}=\phi_{1} p_{3} \Pi_{\alpha}$ touches $\Pi_{\alpha}$ at the points of $B^{n-2}$ for all $\alpha \in[-\pi / 2, \pi / 2]$. In particular, the condition of Lemma 3.2 for $p_{2}$ is fulfilled for $\bar{p}_{3}$. 
Proof. At first note that if the hypothesis of touching is fulfilled for some dense set of $\alpha \in[-\pi / 2, \pi / 2]$, it will be fulfilled for all $\alpha$ from this interval. So, we will try to obtain its fulfillment for a countable dense set of values, namely for the set $\{\alpha=\pi d\}$, where $d$ is a dyadic rational and $|d| \leq 1 / 2$.

Enumerate these numbers into a sequence $\alpha_{k}$ (starting with the four given values of $\alpha$ ), and begin constructing a countable sequence of $\varepsilon_{k}$-isotopies $\phi^{(k)}$, each of which is identical on the $\varepsilon_{k}$-neighborhood of $B^{n-2}$ and achieves the touching condition for the homeomorphism $\phi^{(k)} \cdots \phi^{(1)} p_{3}$ at the points of $B^{n-2}$ for every next $\alpha_{k}$ without loosing this property for the preceding $\alpha_{i}$.

In this construction, independently from the preceding steps, one may take the numbers $\varepsilon_{k}$ arbitrarily small. Hence the sequence of isotopies $\phi_{t}^{(k)} \cdots \phi_{t}^{(1)}$ will tend to an isotopy, and in the limit the touching condition will be fulfilled for all $\alpha \in[-\pi / 2, \pi / 2]$.

It is sufficient to describe one step of the construction, say, for values of $\alpha$ given in the lemma. Next steps are absolutely analogous.

Let us show how to obtain this touching condition for $\alpha=\pi / 4$. The construction of the isotopy in demand takes several steps.

Step 1. Note that by applying the arcwise homeomorphisms one can get that the images of $\Pi_{\alpha}$ touch $\Pi_{\alpha}$ on one side; for example, that $p_{3} \Pi_{0}$ touches $\Pi_{0}$ and lies in $Q(0, \pi / 2)$, and $p_{3} \Pi_{-\pi / 4}$ touches $\Pi_{-\pi / 4}$ and lies in $Q(-\pi / 4,0)$. (Certainly, it is sufficient for each touching condition to be fullfilled in a small neighborhood of $B^{n-2}$.) Let us show this for $p_{3} \Pi_{0}$.

Note that $\Pi_{3 \pi / 8}$ and $\Pi_{\pi / 4}$ lie in a small neighborhood of $B^{n-2}$ between $p_{3} \Pi_{\pi / 2}$ and $p_{3} \Pi_{0}$ and do not intersect them except for $\mathbb{R}^{n-2}$.

Construct an arcwise homeomorphism $\tau^{\prime}$, identical outside $Q(0,3 \pi / 8)$, that sends $\Pi_{\pi / 4}$ to the fence $S_{0}$ touching $\Pi_{0}$. Replace $p_{3}$ by $\tilde{p}_{3}=\tau^{\prime} p_{3}$. All the hypotheses of the lemma remain true, but now $\widetilde{p}_{3} \Pi_{0}$ lies between the fence $S_{0}$ and $\Pi_{-\pi / 16}$ (in a small neighborhood of $\left.B^{n-2}\right)$, since $\tilde{p}_{3} \Pi_{0}$ touches $\Pi_{0}$.

Construct now an arcwise homeomorphism $\tau^{\prime \prime}$, identical outside the domain in $\mathbb{R}_{+}^{n}$ bounded by $S_{0}$ and $\Pi_{-\pi / 8}$, that sends $\Pi_{\pi / 16}$ to $\Pi_{0}$. The lemma's hypotheses remain true after replacing $\tilde{p}_{3}$ by $p_{3}^{\circ}=\tau^{\prime \prime} \tilde{p}_{3}$, but then $p_{3}^{\circ} \Pi_{0}$ lies between $\Pi_{0}$ and $S_{0}$, that is, on one side of $\Pi_{0}$.

Thus from the very beginning we may suppose that $p_{3} \Pi_{0}$ lies in $Q(0, \pi / 4)$ and touches $\Pi_{0}$ as well as, analogously, that $p_{3} \Pi_{-\pi / 4} \subset Q(-\pi / 4,0)$ and touches $\Pi_{-\pi / 2}$. Moreover, $p_{3} \Pi_{\pi / 2} \subset Q(\pi / 4, \pi / 2)$ and touches $\Pi_{\pi / 2}$ as well as $p_{3} \Pi_{-\pi / 2} \subset Q(-\pi / 2,-\pi / 4)$ and touches $\Pi_{-\pi / 2}$.

Step 2. Construct an arcwise homeomorphism $\tau_{1}$, identical outside $Q(0, \pi / 2)$, that sends $\Pi_{\pi / 4}$ to the fence $S_{1}$ touching $\Pi_{0}$, closely to $\Pi_{0}$ so that $p_{3} S_{1} \subset Q(0, \pi / 4)$. Let $t_{1}=p_{3} \tau_{1} p_{3}^{-1}$. Then $t_{1}=1$ outside $p_{3} Q(0, \pi / 2)$ and $t_{1} p_{3} \Pi_{\pi / 4} \subset Q(0, \pi / 4)$. Let $p_{3}^{\prime}=t_{1} p_{3}$.

Step 3. Construct now a fence $S_{2}$ touching $\Pi_{-\pi / 4}$ closely so that $p_{3}^{\prime} S_{2}$ lies in $Q(-\pi / 4,0)$.

Let $\tau_{2}$ be an arcwise homeomorphism identical outside $Q(-\pi / 4, \pi / 4)$ that sends $\Pi_{0}$ to $S_{2}$. Let $t_{2}=p_{3}^{\prime} \tau_{2} p_{3}^{\prime-1}$. Then $t_{2}=1$ outside $p_{3}^{\prime} Q(-\pi / 4, \pi / 4), t_{2} p_{3}^{\prime} \Pi_{0} \subset Q(-\pi / 4,0)$, and $t_{2} p_{3}^{\prime} \Pi_{\pi / 4} \subset Q(0, \pi / 4)$. Let $p_{3}^{\prime \prime}=t_{2} p_{3}^{\prime}$. 
Step 4. Construct a fence $S_{2}$ that touches $\Pi_{0}$ and separates $\Pi_{0}$ from $p_{3}^{\prime \prime} \Pi_{\pi / 4}$. Construct also another fence $S_{2}^{\prime}$ touching $\Pi_{\pi / 4}$ and lying in $Q(0, \pi / 4)$. Let $\tau_{3}$ be an arcwise homeomorphism, identical outside of $Q(0, \pi / 4)$, that sends $S_{2}$ to $S_{2}^{\prime}$. Then $p_{3}^{\prime \prime \prime}=\tau_{3} p_{3}^{\prime \prime} \Pi_{\pi / 4}$ lies in the domain between $\Pi_{\pi / 4}$ and $S_{2}^{\prime}$, that is, it touches $\Pi_{\pi / 4}$ on one side.

Step 5. Consider now the homeomorphism $\bar{p}_{3}=t_{2}^{-1} \pi_{3}^{\prime \prime \prime}=t_{2}^{-1} \tau_{3} t_{2} t_{1} p_{3}$. It is clear that $\bar{p}_{3}=p_{3}$ on $\Pi_{-\pi / 2} \cup \Pi_{\pi / 2} \cup \Pi_{-\pi / 4} \cup \Pi_{0}$ and that $\bar{p}_{3} \Pi_{\pi / 4}$ touches $\Pi_{\pi / 4}$ on one side. Since $\bar{p}_{3} \cdot p_{3}^{-1}$ is identical on $\Pi_{-\pi / 2} \cup \Pi_{\pi / 2}$, there is an isotopy $\phi_{t}$, identical outside $Q(-\pi / 2, \pi / 2)$ and such that $\phi_{0}=1, \phi_{1}=\bar{p}_{3} p_{3}^{-1}$, that is, $\bar{p}_{3}=\phi_{1} p_{3}$.

It is clear that one can make this isotopy $\varepsilon$-small and identical outside the $\varepsilon$-neighborhood of $B^{n-2}$ for any given $\varepsilon$. All conditions of the lemma are satisfied.

Lemma 3.4. Let a homeomorphism $p_{4}: \mathbb{R}^{n} \rightarrow \mathbb{R}^{n}$ be identical on $\mathbb{R}^{n-2}$ such that $p_{4} Q_{k} \subset Q_{k}$, $0 \leq k \leq 3$ where $Q_{0}=Q[-\pi / 8, \pi / 8]$ and $Q_{i}, 1 \leq i \leq 3$ are obtained from $Q_{0}$ by consecutive turns by $90^{\circ}$ counter-clockwise (from $x^{n-1}$ to $x^{n}$ ).

Then for every $\varepsilon>0$ there exists a $\varepsilon$-isotopy $\psi_{t}: \mathbb{R}^{n} \rightarrow \mathbb{R}^{n}$, identical on $\mathbb{R}^{n-2}$ and outside a given neighborhood of $B^{n-2}$, and such that in a smaller neighborhood of $B^{n-2}$ the homeomorphism $\bar{p}_{4}=\psi_{1} p_{4}$, restricted to $\mathbb{R}_{+}^{n}$, fulfills the conditions of Lemma 3.3 for $p_{3}$.

The proof of this lemma follows form an evident construction with arcwise homeomorphisms.

First of all, one may assume, as in the proof of the preceding lemma, that in a neighborhood of $B^{n-2}$ the images of $\Pi_{\alpha_{k}}$ for $\alpha_{k}=k \pi / 2,0 \leq k \leq 3$ touch $\Pi_{\alpha_{k}}$ at points of $B^{n-2}$ and on the wishful side of $\Pi_{\alpha_{k}}$.

Indeed, let $\Pi_{ \pm \pi / 4}$ and $\Pi_{ \pm 3 \pi / 4}$ remain immovable. Move the semiplanes $\Pi_{+\pi / 8}$ and $\Pi_{-\pi / 8}$ by an arcwise homeomorphism $\sigma_{0}$, that is identical outside $Q[-\pi / 4, \pi / 4]$, to $\Pi_{0}$ and to a fence $S_{0}$ that touches $\Pi_{0}$, respectively.

By the same way, let an arcwise homeomorphism $\sigma_{1}$, identical outside $Q[\pi / 4,3 \pi / 4]$, move the semiplane $\Pi_{\pi / 2+\pi / 8}$ to $\Pi_{\pi / 2}$ and $\Pi_{\pi / 2-\pi / 8}$ to a fence $S_{1}$ touching $\Pi_{\pi / 2}$. Let $\sigma_{2}$ be an arcwise homeomorphism, identical outside $Q[3 \pi / 4,5 \pi / 4]$, that sends $\Pi_{-\pi-\pi / 8}$ to $\Pi_{-\pi}$ and $\Pi_{-\pi+\pi / 8}$ to a fence $S_{2}$ touching $\Pi_{-\pi}$.

At last, let $\sigma_{3}$ be an arcwise homeomorphism that is identical outside $Q[-3 \pi / 4,-\pi / 4]$ and sends $\Pi_{-\pi / 2-\pi / 8}$ and $\Pi_{-\pi / 2+\pi / 8}$ to $\Pi_{-\pi / 2}$ and to a fence $S_{3}$ touching $\Pi_{-\pi / 2}$, respectively.

Let $\sigma$ be a homeomorphism that in each fourth-space, limited by semiplanes $\Pi_{ \pm \pi / 4}$ and $\Pi_{ \pm 3 \pi / 4}$, coincides with the corresponding $\sigma_{k}, 0 \leq k \leq 3$. Construct an arcwise homeomorphism $\tau: \mathbb{R}^{n} \rightarrow \mathbb{R}^{n}$ that sends the domain between $\Pi_{\pi}$ and $S_{2}$ to the domain between $\Pi_{-\pi / 2}$ and $S_{3}$ as well as the domain between $\Pi_{-\pi / 2}$ and $S_{3}$ to the domain between $\Pi_{-\pi / 4}$ and a fence touching $\Pi_{-\pi / 4}$. Also, let $\tau=1$ in $Q[-\pi / 8, \pi-\pi / 8]$ and on $\Pi_{ \pm \pi / 4} \cup \Pi_{ \pm 3 \pi / 4}$.

It is clear that $\bar{p}_{4}=\tau \sigma p_{4}$ satisfies the hypothesis of Lemma 3.3 for $p_{3}$. Namely, $\bar{p}_{4} \mathbb{R}_{+}^{n} \subset$ $\mathbb{R}_{+}^{n}, \bar{p}_{4} \Pi_{ \pm \pi / 2}$ touches $\Pi_{ \pm \pi / 2}, \bar{p}_{4} \Pi_{0}$ touches $\Pi_{0}$, and $\bar{p}_{4} \Pi_{-\pi / 4}$ touches $\Pi_{-\pi / 4}$; also, $\bar{p}_{4}$ is isotopic to $p_{4}$ by a small isotopy, since an arcwise homeomorphism is isotopic to the identity and its mesh does not supersede diameters of circles $C_{x}(r)$ and of their images on which it is not identical.

It should be pointed out that the homeomorphism $\tau$, constructed above, is identical on $\Pi_{\pi / 2+\pi / 4}$. 
Lemma 3.5. Assume that there exists a homeomorphism $p_{5}: \mathbb{R}^{n} \rightarrow \mathbb{R}^{n}$ such that $p_{5} Q[-\pi / 8$, $\pi / 8] \subset Q(-\pi / 8, \pi / 8)$.

There is an isotopy $\chi_{t}: \mathbb{R}^{n} \rightarrow \mathbb{R}^{n}$, identical on $\mathbb{R}^{n-2}$ and outside a neighborhood of $B^{n-2}$, such that $\chi_{0}=1$ and $\chi_{1} p_{5}=\bar{p}_{5}$ satisfies the hypothesis of Lemma 3.4 for $p_{4}$ and coincides with $p_{5}$ on $B_{-}^{n-1} \cup p_{5} B_{+}^{n-1}$.

Proof. Consider the 4-sheeted covering $\nu: \mathbb{R}_{2}^{n} \rightarrow \mathbb{R}_{1}^{n}$, branched over the subspace $\mathbb{R}_{1}^{n-2} \subset$ $\mathbb{R}_{1}^{n}$. (It is convenient to indicate distinction between the same objects in the domain and in the image spaces of the covering $\nu$ by means of lower indices.) Denote by $j: \mathbb{R}_{2}^{n} \rightarrow \mathbb{R}_{1}^{n}$ the natural identification of both spaces. Let us concretize the covering by identifying every plane $P_{k x} \subset \mathbb{R}_{i}^{n}$, where $x \in \mathbb{R}_{k}^{n-2}, k=1,2$, with the complex line $\mathbb{C}^{1}$ (xs is the real and $x t$ is the imaginary axes), and by representing $\nu$ as the function $z \mapsto e^{\mathbf{i} \cdot 3 \varphi_{z}} z$, where $z=\rho_{z} e^{\mathbf{i} \varphi_{z}}$. Here $j=v$ on $\mathbb{R}_{2+}^{n-1}$.

According to the hypothesis of Lemma 3.4 the homeomorphism $p_{5}: \mathbb{R}_{1}^{n} \rightarrow \mathbb{R}_{1}^{n}$ is given. Consider the homeomorphism $\tilde{p}_{5}: \mathbb{R}_{2}^{n} \rightarrow \mathbb{R}_{2}^{n}$, covering $p_{5}\left(\nu \tilde{p}_{5}=p_{5} \nu\right)$. We have $v \tilde{p}_{5}=p_{5} j$ on $\mathbb{R}_{2+}^{n-1}$.

Construct now a homeomorphism $\beta: \mathbb{R}_{2}^{n} \rightarrow \mathbb{R}_{2}^{n}$, patching up the covering $p$ so that $\nu \beta=j$ on $Q_{2}[-\pi / 8, \pi / 8]$. Namely, $\beta$ is an arcwise homeomorphism, identical on $Q_{2}[-\pi / 4, \pi / 4]$, that sends $Q_{2}[-\pi / 8, \pi / 8]$ into $Q_{2}[-\pi / 32, \pi / 32]$. (One may analogously redefine $\beta$ on other three quadrants, separated by planes $x^{n}= \pm x^{n-1}$, so that the mapping would remain a covering, but it is not important for us.)

As a result, $\nu \beta^{-1}=j$ on $Q_{2}[-\pi / 8, \pi / 8]$. The homeomorphism $\bar{p}_{5}: \mathbb{R}_{1}^{n} \rightarrow \mathbb{R}_{1}^{n}$, defined by equality $\bar{p}_{5}=j \beta^{-1} \tilde{p}_{5} \beta j^{-1}$, coincides on $Q_{1}[-\pi / 8, \pi / 8]$ with $p_{5}=\nu \beta \beta^{-1} \nu^{-1} p_{5} \nu \beta \beta^{-1} \nu^{-1}$. Moreover,

$$
\bar{p}_{5} Q_{1}[-k \pi / 8, k \pi / 8] \subset Q_{1}[-k \pi / 8, k \pi / 8], \quad 0 \leq k \leq 3 .
$$

So, $\bar{p}_{5}$ satisfies the hypothesis of Lemma 3.4 for the homeomorphism $p_{4}$, as what is required.

Besides, $\bar{p}_{5}$ is isotopic to the homeomorphism $p_{5}$ under isotopy that is identical on $\mathbb{R}_{1}^{n-2}$, since $\bar{p}_{5} p_{5}^{-1}$ is identical on $Q_{1}[-\pi / 8, \pi / 8]$.

\section{Proof of the theorem}

Since the embedding $q_{-}$is topologically flat, it can be extended to a homeomorphism of $\mathbb{R}^{n}$ and so we can assume that $q$ is identical on $B_{-}^{n-1}$. Construct two fences $S_{-\pi}$ and $S_{\pi}$ on two different sides of $B_{-}^{n-1}$, that are touching $\mathbb{R}^{n-1}$ from above and from below and separating $B_{-}^{n-1}$ from $q B_{+}^{n-1}$. Then move them by an arcwise homeomorphism $\tau$, identical on $B_{-}^{n-1}$, onto $\Pi_{-\pi / 8}$ and onto $\Pi_{\pi / 8}$, respectively, and replace $q$ with $\tilde{q}=\tau q$. We obtain $\tilde{q} B_{+}^{n-1} \subset Q(-\pi / 8, \pi / 8)$. Suppose that this is valid for $q$ from the very beginning.

Since $q_{+}$is topologically flat, it can be extended to a homeomorphism $h: \mathbb{R}^{n} \rightarrow \mathbb{R}^{n}$ $\left(\left.h\right|_{B_{+}^{n-1}}=\left.q\right|_{B_{+}^{n-1}} \subset Q(-\pi / 8,+\pi / 8) \subset \mathbb{R}_{+}^{n}\right)$. As $h B_{+}^{n-1} \cap B_{-}^{n-1}=B^{n-2}$, applying as well as above the arcwise homeomorphisms, identical on $B_{-}^{n-1}$, one can wangle $h Q[-\pi / 2, \pi / 2] \subset$ $Q(-\pi / 2, \pi / 2)$. 
Thus the assertion of our theorem now takes the following form.

TheOREM 4.1. Suppose that there is a homeomorphism $h: \mathbb{R}^{n} \rightarrow \mathbb{R}^{n}$, for which $h B_{+}^{n-1} \subset$ $Q(-\pi / 8, \pi / 8)$. Then the cell $B_{-}^{n-1} \cup h B_{+}^{n-1}$ is topologically flat. More precisely: there exists a homeomorphism $\bar{h}: \mathbb{R}^{n} \rightarrow \mathbb{R}^{n}$, identical on $B_{-}^{n-1}$, that coincides with $h$ on $B_{+}^{n-1}$.

The proof of this statement follows from the above sequence of lemmas as follows:

First, having constructed an arcwise homeomorphism $\gamma$, identical on $\mathbb{R}_{-}^{n}$, that sends $\Pi_{-\pi / 8}$ and $\Pi_{\pi / 8}$, respectively, to fences $S_{-}$and $S_{+}$, touching $\Pi_{0}$ from below and from above and separating $\Pi_{0}$ from $h^{-1} \Pi_{-\pi / 8}$ and $h^{-1} \Pi_{\pi / 8}$, we may replace $h$ by a homeomorphism $h y$ that coincides with $h$ on $B_{+}^{n-1}$ and moves $Q[-\pi / 8, \pi / 8]$ into $Q(-\pi / 8, \pi / 8)$. Then we obtain a homeomorphism satisfying the hypotheses of Lemma 3.5 for $p_{5}$.

By Lemma 3.5 we obtain a homeomorphism that coincides with the given $h$ on $B_{+}^{n-1}$ and satisfies the hypotheses of Lemma 3.4 for the homeomorphism $p_{4}$. By Lemma 3.4 we can construct a homeomorphism $\bar{p}_{4}$ that satisfies the condition for $p_{3}$ from Lemma 3.3, is isotopic to $p_{4}$, and is identical on $\Pi_{3 \pi / 4}$ by its construction.

Denote by $D$ the semiball in $\Pi_{3 \pi / 4}$, bounded by $B^{n-2}$, and by $\gamma$ the arcwise homeomorphism, constructed in Lemma 3.4, that is identical on $D$. Evidently, the cell $B_{-}^{n-1} \cup h B_{+}^{n-1}$ is topologically flat if and only if the same is true for the cell $D \cup h B_{+}^{n-1}$, if and only if it is so for $D \cup \gamma h B_{+}^{n-1}$, and if and only if it is so for $B_{-}^{n-1} \cup \gamma h B_{+}^{n-1}$, because these cells are obtained one from another by the application of some (arcwise) homeomorphisms of the space.

So, it is sufficient to prove that the cell $B_{-}^{n-1} \cup p_{3} B_{+}^{n-1}$ is topologically flat, where $p_{3}$ is the embedding given in Lemma 3.3.

According to Lemma 3.3 we can replace $p_{3}$ by an embedding, isotopic to $p_{3}$ under the isotopy, identical on $B_{-}^{n-1}$, that satisfies the conditions of Lemma 3.2. This isotopy sends the cell $B_{-}^{n-1} \cup p_{3} B_{+}^{n-1}$ to the cell $B_{-}^{n-1} \cup \bar{p}_{3} B_{+}^{n-1}=B_{-}^{n-1} \cup p_{2} B_{+}^{n-1}$ and we have to prove that the latter is locally flat. But this is the assertion of Lemma 3.2.

The theorem follows.

\section{Acknowledgment}

The work was supported by Grant 03-01-00705 from RFBR.

\section{References}

[1] J. C. Cantrell, Separation of the n-sphere by an $(n-1)$-sphere, Transactions of the American Mathematical Society 108 (1963), 185-194.

[2] A. V. Chernavsky, Isotopy of elements and spheres in $n$-dimensional space with $k<\frac{2}{3} n-1$, Doklady Akademii Nauk SSSR 158 (1964), no. 1, 62-65 (Russian).

[3] __ Homeomorphisms of Euclidean space and topological imbeddings of polyhedra in Euclidean spaces. III, Mathematics in USSR Sbornik 75 (1968), no. 2, 264-295.

[4] , The k-stability of homeomorphisms and the union of cells, Doklady Akademii Nauk SSSR 180 (1968), no. 3, 1045-1047 (Russian).

[5] R. H. Fox and E. Artin, Some wild cells and spheres in three-dimensional space, Annals of Mathematics. Second Series 49 (1948), no. 4, 979-990.

[6] L. V. Keldyš, Topological imbeddings into Euclidean space, Trudy Matematicheskogo Instituta Imeni V. A. Steklova 81 (1966), 184 (Russian). 
[7] R. C. Kirby, The union of flat $(n-1)$-balls is flat in $\mathbb{R}^{n}$, Bulletin of the American Mathematical Society 74 (1968), 614-617.

[8] A. B. Sosinskiŭ, Imbedding of a $k$-dimensional element in $E^{n}$, Doklady Akademii Nauk SSSR 139 (1961), no. 6, 1311-1313 (Russian).

A. V. Chernavsky: Institute of the Information Transmission Problems, Russian Academy of Sciences, Bolshoy Karetny per. 19, Moscow 117 447, Russia

E-mail address: chernav@iitp.ru 


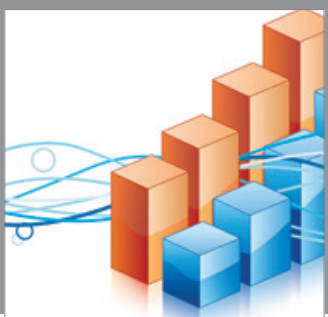

Advances in

Operations Research

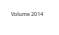

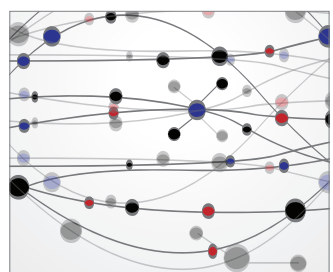

\section{The Scientific} World Journal
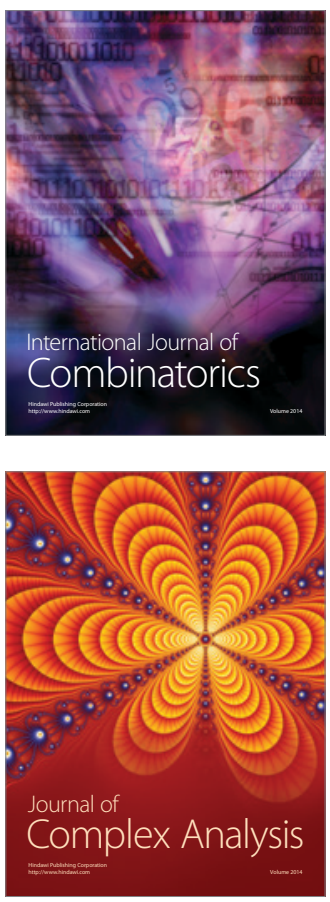

International Journal of

Mathematics and

Mathematical

Sciences
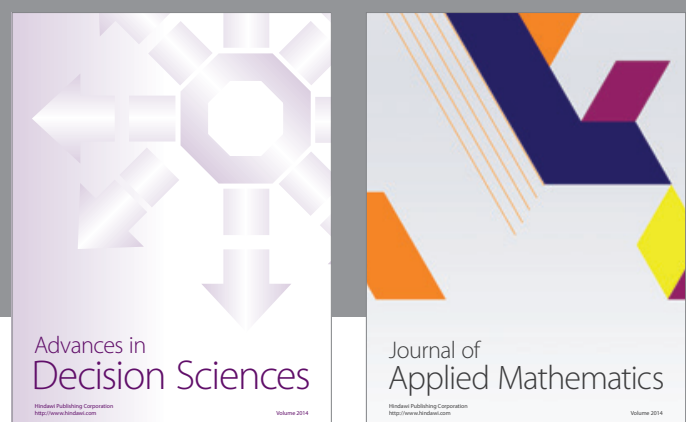

Journal of

Applied Mathematics
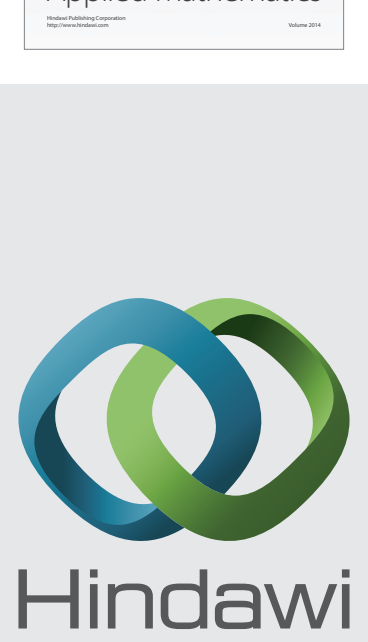

Submit your manuscripts at http://www.hindawi.com
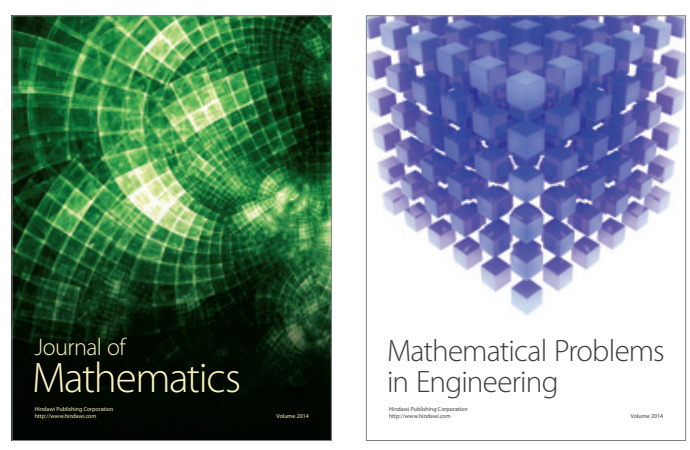

Mathematical Problems in Engineering
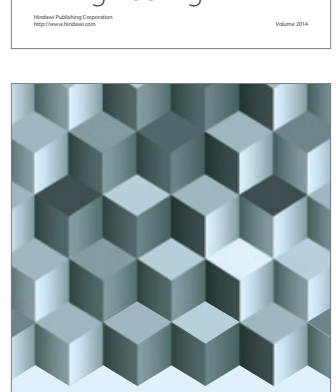

Journal of

Function Spaces
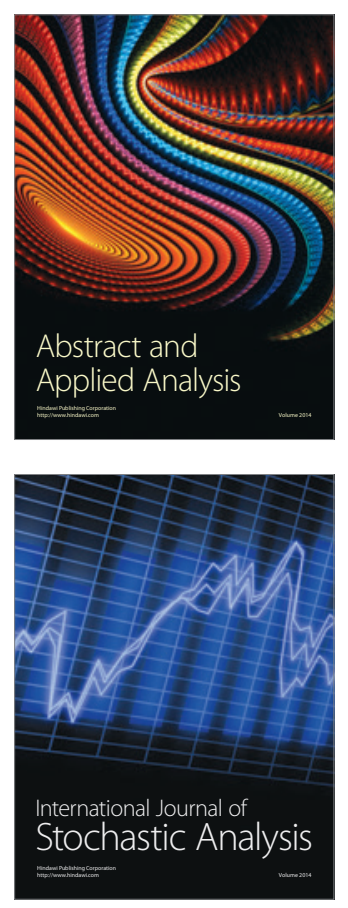

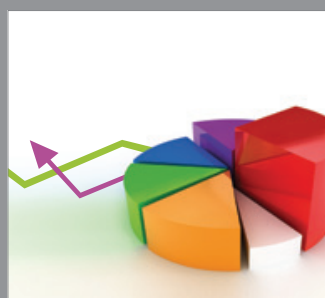

ournal of

Probability and Statistics

Promensencen
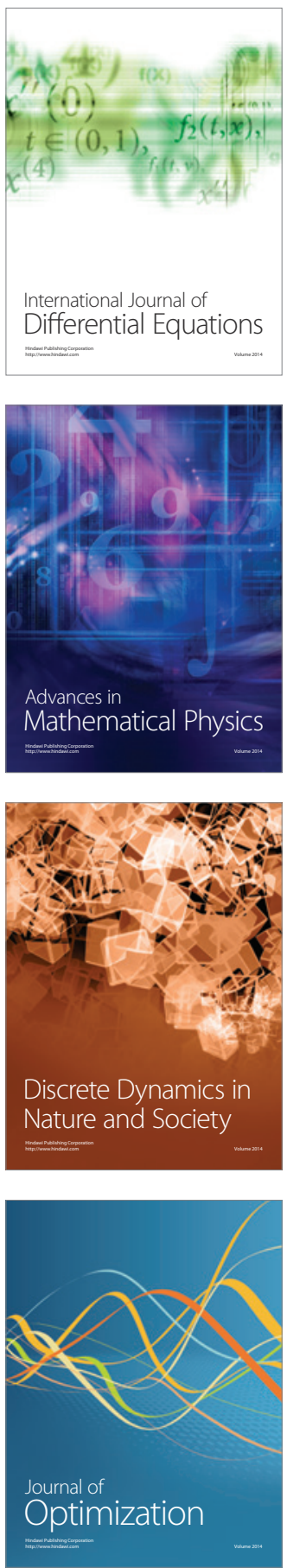